\title{
LA VALORACIÓN DEL CAPITAL CULTURAL EN ESCOLARES CON SITUACIONES DE VULNERABILIDAD SOCIAL
}

\section{EVALUATION OF CULTURAL CAPITAL OF STUDENTS IN SOCIAL VUNERABILITY SITUATIONS}

\author{
Eduardo José Weffer Villarroel ${ }^{1}$ \\ Universidad Pedagógica Experimental Libertador - UPEL
}

\section{Resumen}

La educación, como un acto de vital importancia en la sociedad, constantemente esta inmersa en momentos históricos, sociales y contextuales que sin duda alguna buscan ajustarla a los requerimientos y necesidades que se viven y experimentan. Es así que en los actuales momentos estamos sumergidos en nuevos caminos investigativos y de aportes teóricos, filosóficos y pedagógicos que buscan posicionar la tarea de educar en un elemento trascendental de humanización ante escenarios de crisis sociales y otros aspectos degradantes y divisorios en la sociedad de hoy. Resulta evidente además que la formación de las nuevas generaciones amerita estar enmarcada dentro de dinámicas sociales que permitan generar cambios positivos y consolidar así una sociedad cada día más justa, donde se resalte lo subjetivo y humano, la igualdad de condiciones, entre otros intereses consagrados como derechos humanos. En el presente ejercicio investigativo y hermenéutico de tipo documental, se mostraran inquietudes, reflexiones y posibles sugerencias que de una u otra manera buscan dar un aporte a la educación en los actuales momentos, específicamente en espacios sociales y educativos donde las condiciones de vida y convivencia no son las más adecuadas, pues en los mismos se enfrentan un sinfín de situaciones que desde la educación se han encontrado desasistidas. Me permito entonces a través de este texto el intento de posibilidades en las formas y maneras de encontrarnos con nosotros como docentes y con nuestros estudiantes para así asumir, apreciar, propiciar y atender el acto de educar como una posibilidad de formación para la vida.

Palabras claves: Educación; Condición Humana; Pedagogía; Formación para la Vida.

\footnotetext{
${ }^{1}$ Profesor en Especialidad Educación Integral. UPEL-IPM. Magister en Educación Mención Educación Superior UPEL-IPM. Participante de Doctorado en Educación UPEL-IPM. Docente adscrito al Departamento de Pedagogía y Practica Profesional en el Área de Psicología y Orientación Educativa de la Universidad Pedagógica Experimental Libertador Núcleo Monagas- Venezuela. Investigador A1 PEII 2015.
} 
Abstract

Education, as an act of importance in society, is constantly immersed in historical, social and contextual moments that undoubtedly seek to adapt it to the requirements and needs experienced. Thus, we are currently immersed in new investigative paths and theoretical, philosophical and pedagogical contributions which seek to position the task of educating within a transcendental element of humanization in the face of scenarios of social crises and other degrading and determinant aspects of today's society. It is also evident that the formation of the new generations deserves to be framed in social dynamics that allow to generate positive changes and to consolidate a more just society every day, where the subjective and the human, the equality of conditions, among other interests consecrated as human rights prevail. The present documentary-type investigation and hermeneutic work will present possible concerns, reflections and suggestions that contribute to education in actuality, especially within the social and educational spaces where the conditions of life and coexistence are not the most appropriate, because that is where one faces with an infinite number of situations, starting with education, that have been neglected. In this way, through this text we search for possibilities within the role of the teacher and with the students, to appreciate, promote and understand the act of educating as a chance of life coaching.

Keywords: Education; Human Condition; Pedagogy; Life Coaching.

\title{
INTRODUCCIÓN
}

\begin{abstract}
Existir humanamente es aprender a mantener la tensión y, al mismo tiempo, el hiato, entre vida y mundo. (De ahí la dificultad de vivir, sin duda, así como de la dificultad de educar.) En otras palabras, si bien es verdad que vivir es iniciar nuevos trayectos, al mismo tiempo, lo que comenzamos jamás es nuevo del todo, nunca somos completamente dueños de nuestras vidas, porque jamás podemos desembarazarnos del mundo (MÉLICH).
\end{abstract}

Desde tiempos antiguos la educación ha sido y es un elemento de mucha importancia con efectos transcendentales en la sociedad, sobre todo en todas las dimensiones del ser humano, pues la misma da cuenta de las acciones y desenvolvimientos de las personas, además de sus formas y manera de vivir y convivir, al igual que percibir y reconocer sus verdades, potencialidades y realidades a través de esquemas culturalmente asumidos desde su contexto de hábitat e historias de vida.

Es así que la escuela o institución educativa siempre esta presente dentro de los contextos sociales tales como urbanismos, vecindades, comunidades entre otros, las cuales son planificadas y están ubicadas geográficamente de manera privilegiadas, con acceso a vialidades y otros servicios básicos que de una u otra manera permiten una vida cómoda, agradable, segura y acorde.

De la misma manera, -la escuela- esta inmersa en espacios sociales que son la contraposición de lo antes mencionado, es decir son entornos de convivencia cotidiana 
caracterizados por ser de difícil acceso, muchas veces improvisados y con el disfrute carente de servicios básicos como agua, electricidad, aseo entre otros aspectos que dan cuenta de un sinfín de "carencias" en servicios públicos, en lo nutricional, en la salud, en principios ciudadanos y familiares, en lo que a seguridad y tranquilidad se refiere, y por ende en aspectos culturales, educativos, académicos siendo entonces la institución educativa un ente y espacio de encuentro de grupos y sub grupos de infantes o personas "socialmente etiquetadas, diferenciadas y legitimadas", con distintos tipos de culturas y modos de ver y entender el mundo.

Ante ese escenario la escuela dentro de las funciones socialmente establecidas debe ocupar una labor formativa que permita la superación de las realidades antes mencionadas cuando estas no convienen en lo social y educativo- o sencillamente dar continuidad de la misma por elección y apatía de los docentes que laboran en dichos espacios sociales los cuales pudieran asumir una posición desinteresada de posibilitar transformaciones sociales, mostrándose -la escuela- despreocupada y pasiva ante mecanismos sociales visibles y ocultos que dan origen y continuidad de la reproducción de dichas culturas y realidades sociales, al respecto Bourdieu (1997) manifesta "los mecanismos, extremadamente complejos, a través de los cuales la institución escolar contribuye (insisto sobre este término) a reproducir la distribución del capital cultural y, con ello, la estructura del espacio social. (p.33).

Siendo este panorama antes indicado, causas de señalamientos y culpas a la escuela como ente de reproducción, colocándola en una situación y atención en exigencias de aspectos que no brinda, que no enseña, que no se interesa y por ende son poco relevantes para la formación para la vida, quedando la misma como un ente que no considera lo que verdaderamente falta, lo que es importante y vital, lo que realmente nutre $y$ da vida $y$ promueve un futuro mejor a esos niños, adolescentes y jóvenes, sino se concentra en lo que hay que exigir y cumplir en términos curriculares y académicos originando de alguna manera situaciones de desigualdad social.

Ante tal situación lo preocupante y vital de la escuela, sobre todo, aquella que esta adentrada en espacios socialmente vulnerables es la de recuperar el reconocimiento del educando como ser humano y de la misma manera identificar los mecanismos de funcionamiento que de una $u$ otra forma conducen a la permanencia condiciones de vida inhumanas, a formas de pensar y de percibir realidades de manera limitada y carente, y a 
través de posibles acciones que desde la escuelas se emprendan permita frenar, erradicar o minimizar la eficacia de tales mecanismos -evidentes y ocultos- en dichos espacios, ante tal escenario, se hace urgente, hacer conciencia de ellos y asumir las responsabilidades que le corresponde a la escuela como ente de formación para la vida en lo que a lo cultural y social se refiere.

Además, se hace necesario reflexionar y cuestionar-nos- como docentes, para servirnos y ser partícipe de prácticas pedagógicas que evidencien el despojo de esas estructuras socialmente establecidas que de una $u$ otra manera dan continuidad a la permanencia de esa situación sociocultural, por lo tanto es necesario emprender nuevos caminos que permitan hacer del acto educativo y la apropiación de saberes, un hecho de indudable calidad, pero calidad humana, calidad innovadora, calidad de integración e inclusión, calidad en lo cortés, en lo creativo, en lo lúdico, armónico y agradable, entre otros elementos que verdaderamente favorecen en intencionalidades humanizadoras de la sociedad y esto es posible a través de la Educación.

La presente ponencia se realiza con el propósito de posibilitar un ejercicio de análisis, reflexión, discusión y argumentación sostenida en ideas desde una posición hermenéutica para aproximarnos teóricamente en los supuestos que ocasiona la aparición del fenómenos tales como la reproducción de condiciones de vulnerabilidad social y la desigualdad educativa desde la escuela adentrada en espacios sociales vulnerables y disponernos en sentidos que inviten y promuevan su erradicación, esto como iniciativa para repensar y fortalecer El Sistema Educativo Venezolano en sus políticas y en las acciones de sus actores.

La intencionaloidad de abrir el presente ejercicio investigativo esta justificada en la pertinencia de la temática abordada y los aspectos que a ella se le atribuyen, la misma permite construir y reflexionar en nuevos ideales que amerita el desempeño de la labor docente, pero especialmente para repensar en nuevas intencionalidades formativas desde lo que corresponde a la dimensión integral del acto educativo, apropiarnos de ellas, acogernos y vivenciar maneras distintas y renovadas de ser y de estar desde la tarea de educar en espacios sociales críticos y vulnerables, donde de manera impredecible se dan circunstancias adversas y favorables, bien sea internas desde nuestros estados de ánimos, emociones y pensamientos o externas y contextuales insertando tales aspectos una 
dinámica y condiciones de convivencia y desenvolvimiento familiar, social y económico enmarcada de constantes retos y desafios a superar.

\section{REALIDADES, CONTEXTO SITUACIONAL}

Muchas son las realidades con las que tiene que luchar las instituciones educativas encontradas en espacios comunitarios en situaciones de pobreza crítica y vulnerabilidad social, donde la desigualdad, la exclusión, el quebrantamiento de los derechos humanos, la discriminación, la violencia, el racismo, la subordinación y la naturalización de formas de vivir en situaciones precarias en niveles nutricionales, socioeconómicos, de salud, de condiciones de vida, colocan a la escuela muchas veces de brazos cruzados y en complicidad de lo que en esos contextos se sufre, se siente, se padece, se reclama atención y auxilio oportuno.

Pareciera entonces que ante tales situaciones la escuela que se encuentra cercada de dichas realidades, ejerce una labor educativa con una población o grupos de estudiantes con carencias bien marcadas y evidentes en su hablar, su voz, su gestos, sus miradas, sus prácticas cotidianas, sus actitudes, su corporeidad y otros elementos que dan cuanta de la vida y actuación de los mismos, que según parámetros o estructuras mentales habituadas de manera social, legítimamente establecidas y normatizadas, pareciera ir en contra de lo que corresponde a una cultura aprobada socialmente, debido a que dichas realidades promueven un total vacío y desesperanza, sin visión en la construcción de futuro, de salida de la situación tan frágil que les agobia, de la esfera de violencia que les atormenta y que lo colocan con posibilidades nulas de superación y de un futuro mejor, es entonces importante repensar la idea de educación en estos espacios sociales.

Se hace necesario la reflexión urgente ante escenarios educativos sociales críticos y vulnerables, donde la condición humana este amenazada o sencillamente esta siendo poco valorada. Al respecto Melich (2010) plantea:

Es necesario que recuperemos las dimensiones humanas del espacio y el tiempo, es decir, del habitar precisamente porque no somos ni ángeles ni bestias. Por ello, teniendo en cuenta las profundas modificaciones que han experimentado, es urgente llevar a cabo una reflexión exhaustiva y critica sobre el estado actual del espacio y tiempo en la existencia humana con la finalidad de poder hacer frente al "terror y la tiranía del tiempo", por los cuales, con mucha frecuencia, se encuentran acompañados por la "violencia en y del espacio.(pág. 140). 
Los niños y jóvenes que hoy asisten a los espacios educativos ubicados en comunidades en condiciones socialmente críticas y vulnerables, debido a las múltiples situaciones desfavorables, no les ha permitido apropiarse de una cultura realmente digna del valor y la armonía de la vida, de lo sensible y humano. Dichos espacios educativos están muy evidentes de ausencias de lo afectivo, de la hospitalidad, de la condescendencia, de relaciones filiales fortalecidas y con mucha presencia de rostros desorientados y desanimados entre otros elementos la hace que predomine en ella una esfera incómoda, inadecuada, hostil y de constante conflictos.

Cuestionar dichas realidades y cuestionarnos en nuestra labor como docentes, resulta un desafío que involucra responsabilidad y sensibilidad por favorecer en la condición humana de los estudiantes que habitan y conviven en dichas situaciones, donde se le permita además interrogarse sobre su realidad y ver mas allá para así posibilitar caminos de esperanzas y oportunidades, al respecto Mélich (2008):

El ser humano, en cambio, puede cuestionar el conjunto de la realidad y cuestionarse él mismo, puede sentir curiosidad, ya que es capaz de preguntarse (a menudo, incluso, de angustiarse) a causa del interrogante ¿qué hay más allá de cualquier «más allá»?»... Este «poner en cuestión» es, para bien o para mal, lo que constituye la «(in)humanidad» de la condición humana (p.104).

La formación que se está dando en los espacios socialmente críticos y vulnerables, pareciera que se encuentra signada a miedos, desajustes, situaciones de estrés, terror, a prácticas sociales que no valoran y promueven lo humano, a formas y experiencias de entender, comprender y promover el acto educativo y de formación para la vida entre otros elementos, que siguen atando a los escolares y habitantes de dichos contextos a un pasado y un presente triste y vacío que marcan duramente huellas que en nada consideran las necesidades de superación y vida digna de la persona y que pide a gritos una sensibilidad humana, una educación que nos mueva, que nos sacuda, que nos obligue a emprender acciones desde los espacios educativos que realmente busquen redimensionar y fortalecer la adecuada formación de la población socialmente desfavorecida.

\section{LA OPORTUNA DISTRIBUCIÓN DEL CAPITAL CULTURAL EN LA ESCUELA Y SU INJERENCIA SOCIAL}

Me permitiré hacer énfasis de la conceptualización de Capital Cultural acuñada por primera vez Bourdieu (1977), al referirla estamos señalando una combinación de aspectos y 
elementos que así lo constituyen, entre ellos se denota la acumulación, dominio y exteriorización de conocimientos producto de la acción educativa, es decir es un elemento subjetivo y objetivo de apropiación de cultura en determinado espacio social, que no aparece de imprevisto, que no se transfiere de manera tangible, sino que ha venido cultivándose y alimentándose en distintos grupos de personas o clases sociales de manera individual, colectiva, gradual y continua a través de distintas esferas de socialización e interrelación de la misma con personas y grupos sociales, partiendo a su vez de experiencias que garantiza el acceso a oportunidades de éxito, social, académico y de integración que permita un favorable desenvolvimiento social y laboral dando cuenta en una calidad de vida digna y bienestar en quienes lo poseen. La apropiación de dicho capital en la mayoría de los casos se inicia en el abrigo de la familia y es reforzado, complementado y certificado en la escuela.

Cuando dicho capital es socializado y trasmitido, deriva un proceso de reproducción y ampliación indetenible que consolida además el acceso a una visión de mundo social extenso. Del mismo modo este puede presentarse de tres maneras o formas conocidas como: incorporado, objetivado e institucionalizado que permiten su identificación y apreciación de manera específica según sea el contexto, así lo explica de manera detallada Bourdieu (2001):

El capital cultural puede existir en tres formas o estados: en estado interiorizado o incorporado, esto es, en forma de disposiciones duraderas del organismo; en estado objetivado, en forma de bienes culturales, cuadros, libros, diccionarios, instrumentos o máquinas, que son resultado y muestra de disputas intelectuales, de teorías y de sus críticas; y, finalmente, en estado institucionalizado, una forma de objetivación que debe considerarse aparte porque, como veremos en el caso de los títulos académicos, confiere propiedades enteramente originales al capital cultural que debe garantizar (p.136).

Con la inserción de la conceptualización de Capital Cultural, en investigaciones con fines sociológicos Bourdieu plantea una hipótesis teórica que le permitiera dar cuenta de aspectos de desigualdad en el rendimiento académico de educandos pertenecientes a diferentes grupos sociales, esto con la finalidad de dar cuenta del desempeño escolar exitoso de los niños pertenecientes a clases sociales y sus subconjuntos.

Como aspecto interesante sumado a los elementos que permiten la identificación del Capital Cultural, es el hecho de que el mismo reconoce una incorporación ligada al cuerpo que indica la interiorización de una cultura, es decir, que es derivado por un proceso arduo y consciente de enseñanza y de aprendizaje donde se invierte el tiempo que se amerite, esto 
como una forma de retribución personal de la propia persona que así lo persigue, y que luego pasa a ser "una posesión que se ha convertido en parte integrante de la persona, en habitus. Del "tener" ha surgido "ser". (ob. Cit. p.140)

\section{POSIBILIDADES DE TRANSFORMACIÓN DESDE LA LABOR EDUCATIVA}

Es importante resaltar la valentía que amerita ser docente en espacios sociales críticos y socialmente vulnerables, y mas aun en los actuales momentos de crisis sociales donde la responsabilidad del docente es irreemplazable en aras de posibilitar la renovación y transformación ante la reproducción de miserias, de ruinas, de agites y depravaciones sociales que atentan y destruyen con toda posibilidad de convivencia humana en la sociedad y su influencia en los esquemas de pensamiento del ser humano, es así que la labor educativa es uno de los más elementales actos de amor donde el sentir, hacer y conocer deben permanecer en una dialógica permanente, a fin de posibilitar nuevas miradas y nuevas intenciones educativas, que consoliden y coadyuven en la pertinente formación de las generaciones presentes y futuras sin distinción social alguna.

La concepción filosófica de María Zambrano, nos invita a identificarnos con la filosofía basada en la esperanza y en la mediación educativa, como una práctica constante y permanente que nos lleve a la transformación tanto de manera individual de nuestro ser como en la sociedad, en función de apropiarnos de una original concepción de mundo, donde no reprochemos las experiencias formativas en la cual hemos estado sumergidos, sino que a través que las mismas sean elementos de reflexión y encuentros para educar y re-educar a las personas y guiarlos para que de manera muy subjetiva puedan conquistar su condición humana y verdadera libertad.

Nos sugiere además, promover una "práctica del saber" que origine una razón mediadora y caritativa y permita aliviar la vida de la humanidad como personas que sienten, que padecen, que son de carne y hueso y adecuar los espacios educativos para garantizar dichas finalidades que permiten erradicar torturas o barreras actitudinales que hacen tropezar y oprimen, a fin de hacer valer las diferentes maneras de crecimiento y desarrollo personal de los estudiantes, respetar sus ritmos y formas de aprender sin imposiciones, ni mucho menos invadir su singularidad, propiciando así la integralidad y conexión de diferentes elementos de la interioridad humana de todos los que vivenciamos el acto educativo a diario. 


\section{REFLEXIONES FINALES Y DE APERTURA EDUCATIVA}

Asumir el compromiso, al parecer no es tan fácil, pero la voluntad para iniciarnos en el mismo resulta de mucha importancia para ir conquistando los espacios desasistidos en la eduación en cuanto a lo humano en referencia a las inquitudes y reflexiones presentadas, a fin de asumir y potenciar una educación donde se resalte lo humano del ser, es decir donde tengamos esa sensibilidad por cada uno de nosotros, esa libertad de pensar, esa actitud de cambio ante realidades no óptimas, no agradables, no beneficiosas para el progreso de la sociedad.

Dice Savater (1997) que "hay que nacer para humano, pero solo llegamos plenamente a serlo cuando los demás nos contagian su humanidad" (p. 22). Como "seres humanos" y "docentes" necesitamos estar siempre sumergidos en una constante formación, en un constante aprender, en una permanente inquietud por indagar, transformar y cuestionar, siempre desde la sensibilidad real, resaltando continuamente la idea de educación y formación para la vida.

De acuerdo a Savater, nuestra condición humana es inexistente la culminación de una formación, es decir, nunca debemos de asumir que ya estamos aptos para tal o cual desempeño, de ser así, la educación como acción transformadora y de constante reflexión no tuviera cabida en la actualidad y en el futuro.

Ocuparnos seriamente de la educación sobre todo en espacios críticos y socialmente vulnerables, es asumir nuevas miradas, desde lo místico del acto educativo, es la tarea que debemos emprender, por lo tanto es imprescindible favorecer en nuevas finalidades pedagógicas y de formación, que permita la intervención y accionar estratégico en los espacios de educabilidad (escuelas, instituciones educativas) a fin de propiciar en los niños, jóvenes y aun adultos que se encuentran en procesos formativos, nuevas formas y maneras de encontrarnos, de ubicarnos en la "espaciotemporalidad" de la educación y sociedad, de brindar alternativas de cambio, de fortalecer el pensamiento crítico y reflexivo, de promover y mantener una completa convicción de que a través de la educación es posible una sociedad más fundamentada en el respeto de la integralidad humana y a la ética de la vida plena y feliz, es decir dar otras miradas a la pedagogía que emprendemos, la cual sea:

una pedagogía que rechaza la construcción social de imágenes que deshumanizan al "distinto"; una pedagogía de la esperanza que nos enseñe en nuestra construcción de lo diferente; podernos unir con los diferentes, una pedagogía que nos enseñe que deshumanizando a los "demás", nos 
deshumanizamos a nosotros mismos (FREIRE, (s/f) citado por SANTOS (2003, p. 13).

Ademas asumir de manera activa concepto de comprender es necesario tomar en cuenta elementos características de tal conceptualización, entre ellos el proceso empático, en la cual resalte la intersubjetividad, la apertura, la simpatía; dichos aspectos nos invitan a repensar en cuanto a cómo de manera personal yo comprendo, formulo y me apropio de una práctica pedagógica tomando en consideración dichos elementos, ¿Qué sucede en nuestras aulas de clases y en todos los espacios de Educabilidad? cuando nos apropiamos de una labor educativa generadora de una enseñanza y un aprendizaje ajustada a esta apreciación de comprender.

Bajo esta premisa, es necesario asumir el acto educativo como un encuentro de comprensión donde "el educador necesita del educando así como el educando necesita del educador", en la cual se brinden de posibilidades y experiencias acogedoras, aun cuando la realidad o el contexto donde se dé no sea la más indicada y agradable, el docente en su muestra de vocación al arte de educar, emprende caminos y formas de interactuar, relacionarse y comunicarse con sus estudiantes, teniendo en cuenta siempre la necesidad de colorear, alegrar y maravillar la vivencia de los mismos en su experiencia de aprendizaje, en donde "Educar como arte es un acto de resistencia a los modelos dominantes de pensar, decir, hacer y sentir. Por esto, también es un acto creador que nos forma y nos transforma" (Tellez 2004, p. 2). De manera que éste pueda percibir una óptica de mundo distinta, diferente, siendo él como educando, el primer partícipe del cambio y trasformación de su entorno.

A diario, toda experiencia educativa nos hace encontrarnos en nuestra condición humana, al igual que lo propiciamos en nuestros estudiantes, pues somos el resultado de un tejido de experiencias que a lo largo de nuestras vidas nos marcan, nos forman, nos direccionan a un pensar, a una visión de vida, de mundo, de realidades, pensamientos y sentimientos.

Es así como la vital consideración y valoración de lo "humano" es apreciado como aspecto fundamental en el proceso de transformación y cambio en todo encuentro educativo y más aun para promover y lograr un aprendizaje contextualizado a la cultura, vocabulario, historia de vida de quienes aprenden, relaciones interpersonales y experiencias individuales y colectivas entre otros elementos. De este modo se originan de manera constantes 
relaciones, diálogo y acercamientos para conocer, descubrir, interactuar y convivir en un armónico y grato encuentro pedagógico.

Dicho encuentro debe prevenir la perspectiva individualista en los involucrados, al contrario, está llamado a incentivar el trabajo colaborativo que permita dar oportunidad a todos y cada uno de los que interviene en el mismo en favorecer una experiencia de aprendizaje que promueva el análisis, la comprensión, la aceptación, lo humano, la reflexión de saberes, permitiendo esto un clima armónico y de total confianza, donde el habito sea la permanente interacción y retroalimentación de conocimientos que consientan la construcción de prácticas reales, pertinentes, innovadoras ajustadas a la necesidad de transformación y superación de realidades no favorables y por ende a la formación que reciben y amerita consolidar, reflejándose de esta manera el beneficio colectivo, tanto en los estudiantes como en los docentes.

Como elemento necesario para apropiarnos del verdadero significado del encuentro pedagógico desde "lo humano" es precisamente "el amor". Al referirnos a esta palabra, podemos sentir que la misma posee una multiplicidad de acciones todas en beneficio del prójimo o de la humanidad, las cuáles pueden ser evidenciadas en gran parte de los acciones que emprendemos a diario, de acuerdo a Morín (1998) el amor se define como "Culminación de la unión entre la locura y la cordura" (p.34), Al fijarnos en esta concepción del autor, podemos afirmar que "el amor" es aquello que nos permite despertar una pasión, una energía, un entusiasmo y una persistencia maravillosa, encantadora, bella en lo que a diario estamos llamados a realizar.

Contextualizando la anterior apreciación a la labor pedagógica y transformadora del docente, debemos apropiarnos de esa actitud de pasión, de magnetismo, imaginación, innovación e iniciativa por desenvolvernos arropados de mucho amor para con nuestros estudiantes y compañeros de los entornos formativos, sinceramente nada cuesta mostrar ese amor al prójimo, esa aceptación, ese respeto a su condición de vida, de intereses y aspiraciones y así juntos contribuir a una sana interacción y confrontación de saberes que busquen primeramente el constante descubrimiento de nuevos conocimientos y por ende el desarrollo y la preparación continua hacia la excelencia y calidad humana.

En esa demostración de amor, resaltar la condición humana de cada uno de los actores del acto educativo, especialmente para con los estudiantes, pues muchas veces cometemos errores que marcan y obstaculizan el sano progreso de los mismos porque 
realmente desconocemos sus intereses, aspiraciones, cultura, concepción de vida y de mundo, irrespetando su "unidualidad" es decir un ser plenamente biológico y cultural, un ser original con potencialidades y debilidades que pueden ser encaminadas a mejoras.

Es precisamente al demostrar ese amor, podemos decir que estamos en la capacidad de comprender lo cual según manifiesta Morín (1998) que involucra "un proceso de empatía, de identificación y de proyección, siempre intersubjetiva, la comprensión necesita apertura, simpatía, generosidad" (p.21).

\section{LA INSTITUCIONALIDAD COMO ESPACIO DE INTERRELACIONES SOCIALES}

En cada acción educativa que se genere en la escuela cobran relevancia las interacciones sociales enmarcadas en mediaciones significativas y contextualizadas que permitan el fortalecimiento del Capital Cultural a través de experiencias de aprendizaje transcendentales que no se agoten solo en el mero cumplimiento de tareas o metas académicas, sino que se dé de manera transversal una esfera de sentidos y complejidades que involucran cada una de ellas. En este sentido se evidenciará las condiciones de eficacia de la institucionalidad de la escuela.

La escuela como espacio de formación y de continua interacción social debe favorecer en acciones que garanticen una instrucción académica desde perspectiva más flexible y de la valoración de subjetivaciones, donde los marcos locales de sentido entendiéndolos como las formas de ver e interpretar el mundo, sumado a sus intereses y condiciones sociales de los estudiantes que allí se congregan tenga relevancia, vitalidad y sean de referente en las acciones, roles, tareas y demandas formativas y por ende diaria labor docente, de esta manera se determinará y dará paso a una renovada institucionalidad determinando esto un nuevo funcionamiento de la escuela adentrada en espacios sociales vulnerables.

Las interacciones sociales que se dan y subyacen en toda institución escolar dan origen a la circulación de sentidos en las tareas, acciones y comportamientos que asumen sus actores, de manera específica sus estudiantes, por lo tanto, estas no pueden entenderse ni verse como mecanismos objetivos de precisión, al contrario, la eficacia de la institucionalidad esta en considerar las mediaciones, contenidos e intencionalidades en la cual están implicadas las dichas relaciones sociales y los sentidos y valoración que los estudiantes le conceden, allí generará transcendencia en el raciocinio y cultura del aprendiz . 
Entendiendo además que las complejidades y características particulares que se que derivan de dinámicas de interacción social entre ellas las situaciones imprevisibles, conflictos, complicidades, conjeturas, rumores, presentimientos, resistencias entre otros aspectos requieren de una atención mas allá de la racionalidad científica u objetiva con una visión estándar, es necesaria una mediación intuitiva, de detección, de proyección de múltiples y deferentes posibilidades de superación, transformación y evolución que darán cabida a entendimientos, alianzas, negociaciones, resistencias para que dicha institucionalidad a través de la distribución del capital cultural - entre otras acciones- pueda ejercer el poder de influencia y de esta manera ser agente de renovación y cambio en las relaciones explicitas y ocultas y las actitudes, y estas derivaran comportamientos que de una $u$ otra manera sumaran un sin de efectos positivos a las funciones y objetivos institucionales siendo esto de beneficio individual y colectivo de sus actores.

Colocar solo como referente lo oficial, lo estándar y lo originalmente establecido de la institucionalidad para luego visionarla y redimensionarla con un orden innovador y renovado funcionamiento de la escuela, constituye una necesidad fundamental, para viabilizar el desarrollo de la autonomía de sus estudiantes, la apropiación de un pensamiento critico y la valoración de sus intereses, sentimientos y expectativas de vida, debido, a que inserta en su dinámica educativa y pedagógica una plataforma de interpretación de sentidos y significados desde lo emergente y la incertidumbre que se da en el entorno social donde se ubica y de las realidades culturales, familiares y personales que allí se suscitan.

Partiendo de lo anterior, es imprescindible entonces que los actores responsables de la institucionalidad asuman constantemente disposición en pensar y repensar acciones que al ser ejecutadas permitirán modificaciones necesarias en las estructuras, dinámicas y estrategias de interacción social que ocurren en la escuela a los fines de favorecer en vivencias y experiencias educativas con real y positiva significación, comprensión e interpretación que permitan la oportuna y adecuada distribución del Capital Cultural en le marco de una plataforma simbólica requerida para tal fin.

Por lo anterior, la escuela necesita dar cuenta de un sistema universal que este despejado de relaciones mecanizadas, instrumentalizadas y racionalizadas, que erradique los sesgos en la atención académica y pedagógica que se brinda a los estudiantes en la distribución del Capital Cultural, así como en la evaluación objetiva de los aprendizajes que se derivan de dicha atención. 
Resultaría entonces de gran avance que la escuela asuma una "institucionalidad" deslastrada de programas mecánicos y normas estables de procesos de transmisión y evaluación de conocimientos, además del empleo de reglas de control en las interacciones que se dan con -y entre- los estudiantes, para esto falta mucha disposición y esfuerzos en políticas educativas, desempeño docente y participación de la familia entre otros aspectos influyentes, pues, la escuela en el ejercicio de dicha "institucionalidad", sobre todo aquella que se encuentra adentrada en entornos socialmente vulnerables aun permanece en la imposibilidad de sacudirse de prácticas escolares y mecanismos de distribución de Capital Cultural, donde solo tiene relevancia la formación académica y socialización signada a estándares de fabricación de individuos que dictan estar lejos de una labor educativa que compensación asuntos relacionados a diferencias individuales y colectivas que provienen de sus contextos familiares, socioculturales e intereses personales.

La institucionalidad de la escuela esta llamada a gestionar brechas de distanciamiento entre elementos de formación que persistan en una visión mecanizada, estándar y precisa por tratarse de formación de personas diversas en aspectos culturales, sociales, intelectuales y mas cuando en atención a los desafíos educativos que impone la sociedad de hoy, donde reclama una formación de las nuevas generaciones que posibilite un desarrollo intelectual- emocional esto en el marco de contextos sociales, realidades distintas y complejas. Es necesario entonces recuperar dispositivos de institucionalidad que rompa con la reproducción y cosificación de comportamientos en los estudiantes y posibilite la consideración de las emociones, intereses y expectativas de los mismos a manera de posibilitar su autonomía de pensamientos, de criterios, de actitudes y una independencia cultural.

Negar el anterior planteamiento, es dar continuidad a una "institucionalidad" que se evidencia solo en una mera "apariencia" escondiendo un sinfín de interacciones subyacentes y suprimidas que la colocan como una escuela que no da origen a cambios y transformaciones visibles, mucho menos a transcender en la vida y entorno social que favorezca el presente y futuro de sus estudiantes, no obstante de lograrlo, seria en diminutos niveles o de manera ralentizada, perdiendo así el verdadero sentido de institución.

Hoy por hoy la "institucionalidad" de la escuela parece estar centrada únicamente en una organización instrumental de las interacciones que se dan en ella, es decir, se interesa solo en una cultura de lo disciplinar del saber fragmentado de los conocimientos que si no se 
le suman y consideran aspectos culturales y contextualizados del entorno social donde ocurre el acto educativo en poco transcienden y favorecen a los estudiantes en las dimensiones que conforman el conjunto y el todo de su integralidad como seres humanos en evolución y desarrollo.

\section{REFERENCIAS}

BOURDIEU, P. (1997). Razones prácticas sobre la teoría de la acción. Barcelona: Anagrama.

BECERRA, S. (2012) Educacion en contextos de pobreza: visibilizacion del fenomeno del prejuicio docente.Chile. Universidad Catolica de Temulco.

CASADO, A., \& SANCHEZ-GEY, J. (2007). Filosofia y Educacion en Maria Zambrano. Revista Española de Pedagogia., 545-558.

MELICH, J \& BOIXADER,A. (2010) Los Márgenes de la Moral. Una Mirada Ética de la Educación. Barcelona. GRAO.

MELICH, J.(2008). Antropología, Narrativa y Educación. Barcelona. Universidad Autónoma de Barcelona.

MELICH, J.(2010). Ética de la Compasión. España. Herder.

MORIN, E. ( 1998). Amor, Poesía, Sabiduría. Caracas-Venezuela.: UCV-Faces.

MORIN, E. (2000). Los Siete Saberes Necesarios a la Educacion del Futuro. CaracasVenezuela: UCV-FACES.

SANTOS, M. S. (2003). Pedagogia de la Diversidad. Chile: LOM Ediciones Ltda.

SAVATER, F. (1998). El Valor de Educar. Colombia: Ariel.

TELLEZ, M. (2004). Educacion, Comunidad y Libertad. Notas sobre el educar como experiencia etica y estetica. Revista de Pedagogia. 